\title{
The New Area Subdivision Methods for Producing Shapes of Colored Paper Mosaic
}

\author{
Sang Hyun Seo ${ }^{1}$, Dae Wook Kang ${ }^{1}$, Young Sub Park ${ }^{2}$, and Kyung Hyun Yoon ${ }^{2}$ \\ 1 Department of Image Engineering Graduate School of Advanced Imaging Science, \\ Multimedia, and Film, University of Chung-Ang, DongJak-Gu, Seoul, Korea \\ \{ddipdduk, gigadeath\}@cglab.cse.cau.ac.kr \\ http://cglab.cse.cau.ac.kr \\ 2 Department of CS\&E University of Chung-Ang, DongJak-Gu, Seoul, Korea \\ \{cookie, khyoon\}@cglab.cse.cau.ac.kr
}

\begin{abstract}
This paper proposes a colored paper mosaic rendering technique based on image segmentation that can automatically generate a torn and tagged colored paper mosaic effect. The previous method[12] did not produce satisfactory results due to the ineffectiveness of having to use pieces of the same size. The proposed two methods for determination of paper shape and location that are based on segmentation can subdivide an image area by considering characteristics of image. The first method is to generate a Voronoi polygon after subdividing the segmented image again using a quad tree. And the second method is to apply the Voronoi diagram on each segmentation layer. Through these methods, the characteristic of the image is expressed in more detail than the previous colored paper mosaic rendering method.
\end{abstract}

\section{Introduction}

The trend of current computer graphic techniques, such as radiosity and ray tracing, puts its emphasis on generating a photo realistic image by considering the relationship between light and 3D geometry data in the rendering process. Images obtained through these classical rendering methods do not show any individual character and tend to give a super-realistic expression. Recently, there has been a great deal of interest in NPR (Non-Photorealistic Rendering) techniques. Several computer graphics researchers are studying various ways to simulate conventional artistic expressions such as pen-and-ink illustrations, watercolor, and impressionism. Therefore, the main focus of the NPR methods is to create a special rendering style similar to those created freehand by people. Images created using these methods show an enhanced artistic beauty, more than the images created using traditional computer graphic techniques[4][7].

The colored paper mosaic rendering method, a non-photorealistic rendering method[12], is introduced in order to simulate man-made mosaic work. Various features that produce mosaic effects in mosaic works must be considered. For example, how the paper is attached by hand, the irregular lines of torn paper, and white paper effects that show a cutting plane. These rendering techniques are 
introduced in Seo's paper and will be briefly explained later in this paper. The previous method did not produce satisfactory results due to the ineffectiveness of having to use pieces of the same size. The input image was divided into grids of equal size before the Voronoi sites could be placed, which caused a loss of the characteristics of the image[12].

This paper introduces a method of acquiring a Voronoi diagram by segmenting the input image using the segmentation technique, and then placing the Voronoi site into the area created by splitting the segmented input. The first proposed algorithm locates the Voronoi sites on the location of each tree node by subdividing the segmented area with the quad tree technique. In the second algorithm the Voronoi polygon is generated by the method of applying the Voronoi diagram on each segmented layer. The advantages of this proposed method are that pieces of paper can be aligned along the borderline of the object, and the size of attached paper can be adjusted according to the size of a similar colored image area in order to create a more man-made effect on the produced image.

\section{Previous Works}

The colored paper mosaic rendering method proposed in this paper is the method used to create an image by using pieces of paper torn by hand. Application software that is currently in use, such as Photoshop, provides methods that are similar to the mosaic rendering technique (ex, Mosaic filter, Crystallize filter, Stained Glass filter, Mosaic Tile filter and so on). However, it is difficult to express the colored paper mosaic effect with the filtering methods provided by such graphic tools. The resulting images created using current technology produce fairly artificial and mechanical images, and therefore are not suitable for being applied to create natural images, which is the main focus of NPR.

Hausner introduced the technique that simulates decorative mosaics using CVD(Centroidal Voronoi Diagram), which normally arranges points in a regular hexagonal grid. The direction of the tiles is determined by creating a gradient map after the edges have been determined by placing the tiles[8].

Seo has introduced a new algorithm of automatically simulating the torn colored paper effect with a computer. The shape of the colored-paper is determined by using the Voronoi diagram and the torn colored-paper effect is expressed through the random fractal method[12]. However, in this method, the quality of the resulting image depends largely on the size of the attached paper and the complexity of the input image. Also, the method is limited in its ability to express a natural colored paper mosaic rendering effect due to its limitation of having to use pieces of regular size to express the image.

In this paper, we introduce a new algorithm that determines the shape of the attached paper by taking the edge and area of the object into account by analyzing the input image. 


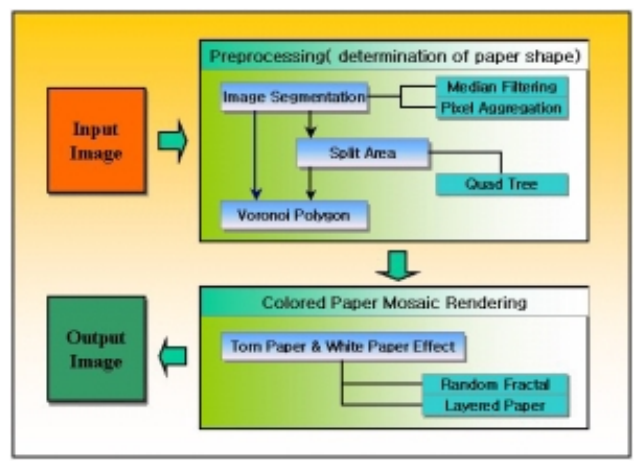

Fig. 1. Mosaic rendering flowchart

\section{The New Area Subdivision Methods for Producing Shapes}

Fig. 1 is the flowchart of our system, which is introduced in this paper. The median filtering and pixel aggregation for the segmentation are processed in the preprocessing stage before the quad tree or the Voronoi diagram is applied. The colored paper mosaic rendering process is applied to each Voronoi polygon that is generated in the preprocessing stage. The details of each process will be illustrated in the following section.

\subsection{Extraction of Features for Determination of Paper Shape}

The object in the input image should be extracted for the colored paper mosaic rendering that expresses the main feature of the image. The edge of the object on the input image can be obtained through extracting the image feature. Edge detection, a method for connecting the detected edge pixels, and the image segmentation method are used to divide the image into objects. In this paper, we use the image segmentation technique. The problem caused by a piece of paper crossing the boundary (edge) of the object that consists of input image can be prevented through image segmenting. The pixel aggregation algorithm[6] has been used as the segmentation method in this paper. The pixel aggregation algorithm is a simple method for segmentation using gray scale images that have been preprocessed through smoothing. This algorithm compares the intensity of the pixel with its neighbor pixels based on the initial coordinate for segmentation. The area is included in the same area if the value for the intensity is smaller than the threshold, and segmented if the value is above the threshold. The edges are used to extract the image feature in determining the polygon to be used to express the mosaic rendering. The effect of putting pieces of the mosaic together manually along the edge of the image can be expressed through this method. Fig. 2 is an example of the segmented image. Fig. 2(a) is input image. Fig. 2(b) 


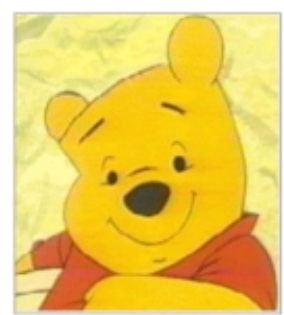

(a) Input image

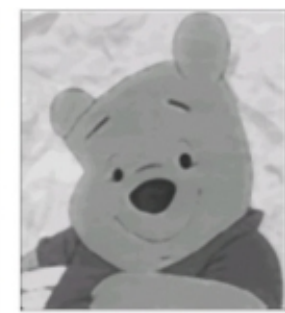

(b) Smoothed Gray Scale Image

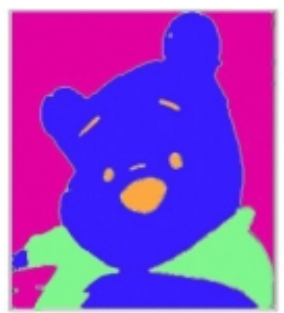

(c) Segmented Image

Fig. 2. Input and segmented image

is smoothed gray scales image. Quarterly segmented images are shown in Fig. $2(\mathrm{c})$.

\subsection{Determination of Paper Shape}

In the work of Seo et al, the Voronoi diagram[9] has been used to generate the shape of an attached paper. The input image is divided into even grid sizes to generate the Voronoi polygon. Random points are generated in each divided area. After creating a Voronoi diagram using these input points, the Voronoi polygons have passed the mosaic rendering stage. In this paper, the Voronoi diagram has also been used to determine the shape of the attached paper. But two new algorithms have been applied to locate the sites of the Voronoi diagram onto the segmented images.

The first method locates the Voronoi sites on each leaf node by subdividing the segmented image using quad tree. In the second method the Voronoi polygon is generated by randomly locating the Voronoi sites into each segmented layer. The Voronoi polygons created through the first method will be smaller in size due to the level of the tree nodes obtained around the edge of the object. This method is effective in expressing the detailed parts of an image by having the effect of illustrating finely torn paper. The second method applies the Voronoi diagram on each layer of the segmented image. Voronoi polygons will be created along the edge (boundary) through the segmentation. Therefore, the feature of the image can be maintained and the borderline formed by curved lines can be expressed precisely.

Voronoi Diagram Based On Quad Tree Subdivision The Voronoi diagram based on Quad Tree[2] further subdivides the segmented image by using the quad tree algorithm. The size of each paper can be controlled for the simulation by increasing or decreasing the subdivision (tree level) rate around the border through the quad tree. The size of each Voronoi polygon can be determined by the size of the node of the quad tree through randomly locating the Voronoi sites for each node. The dense shape of a paper can be expressed more effectively along the border of the image by using this method. 
The root node that contains the whole image is created to acquire the nodes of the quad tree in the combined segmented image. Each node further subdivides itself into four child nodes if the number of segmented layers that it occupies is more than two. The same algorithm is applied repeatedly on the child node obtained through this method until it occupies only a single segmented layer. However, segmentation is disrupted if the size of the node reaches a minimum threshold in order to prevent creating pieces that are too small(Table 1: MINIMUM_SIZE). Table 1 explains a simple pseudo code of the algorithm. Voronoi

Table 1. Pseudo code of quad tree algorithm

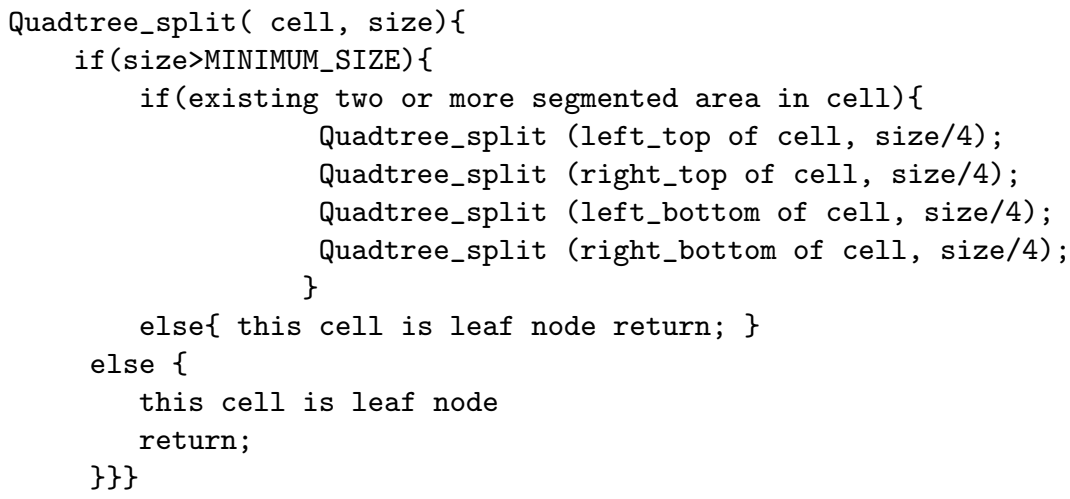

polygons of various sizes are created through random location of Voronoi sites on each node. The shape of each Voronoi polygon determines the shape of the paper. Fig. 3(a) shows the input image. Fig. 3(b) is the subdivided image by quad tree. Finally, Fig. 3(c) is the resulting image of the Voronoi polygon obtained by randomly locating the Voronoi site on the leaf nodes of the quad tree.

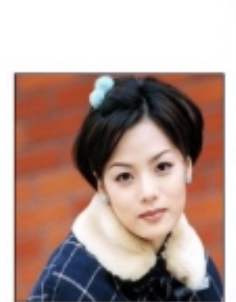

(a) Input image

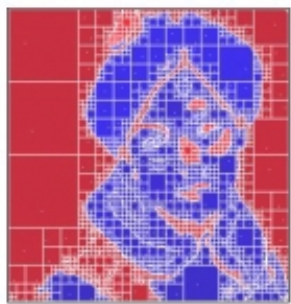

(b) Subdivided image by quad tree

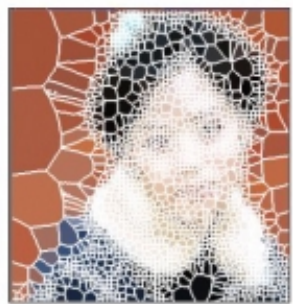

(c) Voronoi polygon

Fig. 3. Voronoi diagram based on quad tree 
Constraint Voronoi Diagram on Each Segmented Layer The algorithm, which is explained in this section, is a method of applying the Voronoi diagram on each segmented layer. In general, when the actual mosaic is created by hand, the boundary of objects in the image and edge of the attached paper are fit to clearly express the objects. However, it is difficult to express the boundary of the objects in the previous colored paper mosaic rendering algorithm because the Voronoi sites are randomly located around the boundary, and generated Voronoi polygons cross the boundary. The polygons created by the Voronoi diagram should not overlap with the boundary of the object so as to express the features of the image. To prevent overlapping, each and every segmented area should be separated and then the Voronoi diagram applied.

This algorithm makes it possible to attach a piece of paper along the boundary of objects in the input image. The Voronoi sites should first be placed randomly and then the Voronoi diagram should be applied on each segmented layer. The Voronoi diagram is applied to each layer of the segmented image to express the boundary of the object. The process of determining the shape of the paper along the borderline is simulated when each Voronoi edge is fitted to the border of the object. The shape of the entire area that the Voronoi diagram should be applied to is either rectangular or regular. Whereas, the shape of the segmented layer that the Voronoi diagram should be applied to is irregular. Now, the boundary of the segmented layer has a curved shape. In this paper, the edge

Table 2. Pseudo code of edge map generating algorithm

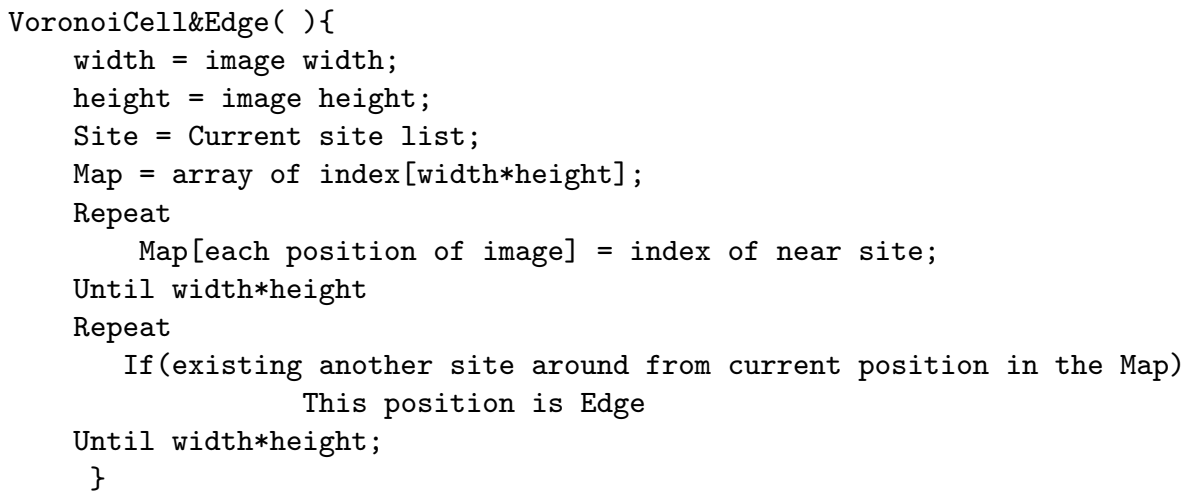

map is used to express the edge of Voronoi polygons generated along the curved boundary. The edge map includes information about the location of pixels and the index of the Voronoi site. The location of pixels is stored in the form of lists, and these lists are approximated as having one or several edges. To construct an edge map, an array of the same size as that of the input image is allocated. Each location of an array stores the index of its closest Voronoi site. The edge 

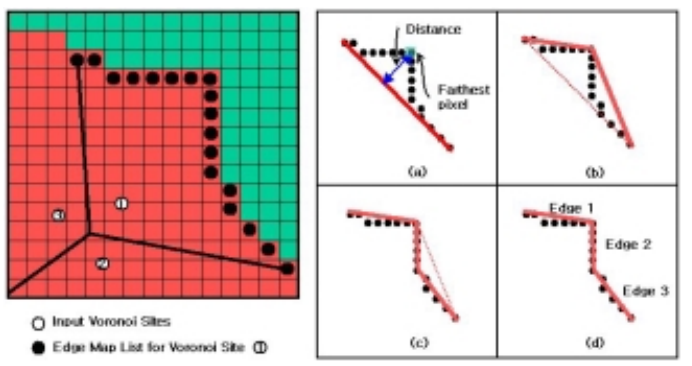

Fig. 4. Edge map for one Voronoi site and approximation of Edge map

map is computed again to determine edge pixel by comparing the number of Voronoi sites stored in the neighbor index. If the number of Voronoi sites stored in the current index is different from that of the neighbor index, the current index is detected as edge pixels. The detected edge pixels are stored as a linked list, which is used to determine the final shape of the paper. By doing so, the edge map for each Voronoi site is completed. Table 2 shows pseudo code of the proposed algorithm.

Fig. 4 shows an edge map for one Voronoi site and the approximation process. The black points in Fig. 4 are the edges map for Voronoi Site 1. The approximation process below is briefly explained.

- Step 1: Initial edge map is approximated as one edge by linking both ends of the list (Fig. 4(a)).

- Step 2: The pixel, which is farthest from the corresponding edge, should be located. If the maximum value of distance is smaller than the defined threshold, the process should cease (Fig. 4(a)).

- Step 3: Based on the located pixel, which has the maximum distance, two sub-edges are constructed (Fig. 4 (b)).

- Step 4: Step 2 is repeated on each newly approximated sub-edge (Fig. 4(c) (d)).

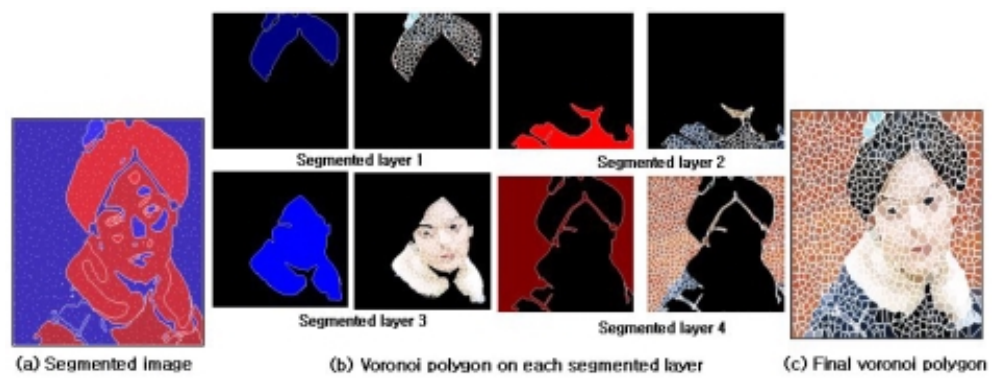

Fig. 5. Constraint Voronoi diagram on each segmented layer 
Fig. 5(a) shows the segmented input image and initial Voronoi sites. In Fig. 5(b), each segmented layer that the Voronoi diagram is applied to is classified (four layers). Finally, Fig. 5 (c) is the Voronoi diagram where four segmented layers are combined.

\subsection{Mosaic Rendering: Torn and White Paper Effect}

Torn Paper Effect. The colored paper mosaic expression is the method of implementing a torn paper effect. The shape of the edge of a torn piece of paper is irregular. A method for adding an irregular height to the straight line connecting the two points on the edges that form the Voronoi polygon is required to express this effect. The random midpoint displacement algorithm, a random fractal[5][10] method, is used to express the irregular shape more naturally in this paper (Fig. 6(a)). Random fractal methods are used for modelling irregular curved lines or curved surfaces that appear in nature. The torn paper effect is implemented by controlling the iteration number and the random amount by the length of the edge line[12].

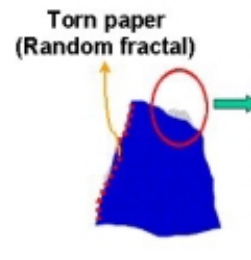

(a) Torn paper

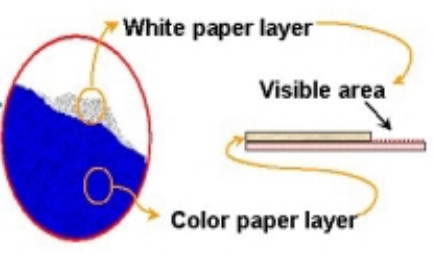

(b) White paper effect

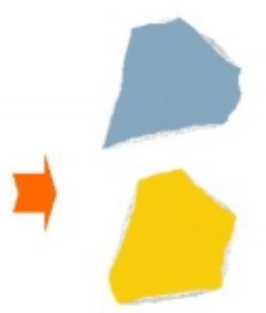

(c) Torn colored paper

Fig. 6. Irregular torn paper and white paper effect

White Paper Effect. A piece of colored paper consists of many different layers of fiber. Therefore, when it is torn apart, it doesn't show an even surface but a rough white surface. In this paper, we assume that a colored paper consists of two different layers. The top layer is the colored layer and the bottom layer is the white paper. Paper colors should represent the image region they cover. Each paper color may be either a point sample form the pixel at the paper's center, or an average of the pixels covered by the paper. The size and the shape of each layer of paper using the random midpoint displacement algorithm are different due to its randomness. With the continued process of attaching these random piece of paper, there comes a case when the white layer is larger than the colored layer, which is the method used to express the white paper effect of the torn paper (Fig. 6(b) Fig. 6(c)) shows the torn colored paper effect created using the proposed method [12]. 


\section{Conclusion and Future Works}

A new algorithm to more naturally determinating the shape of colored paper used for rendering has been introduced in this paper. The image has been classified through segmentation algorithm according to objects. A method of applying the Voronoi diagram on the quad tree, and a method of applying the Voronoi diagram directly onto each segmented layer have been implemented to determine the shape of the colored paper. In the case of the method using the quad tree, the rendered image is similar to actual hand-made mosaic work because smaller pieces of paper are used around the edge of the object while larger pieces of paper are used in the rest of the area. With respect to the Voronoi diagram method based on segmented layer, the boundaries of the object are expressed more clearly. However, The difference in the size of each paper cannot be thoroughly expressed.

These two methods propose that the pieces of paper can be aligned along the border line of the object, and that the size of attached paper can be adjusted according to the size of a similar colored image area to create a more manmade effect on the produced image. Fig. 7 compares the resulting mosaic images created using the basic Voronoi diagram and the methods proposed in this paper. It is evident that the boundary of the image is expressed more clearly in Fig. 7(b) and that the image in Fig. 7(c) expresses the different size of the pieces more clearly, although the boundary is not as clear.

The physical effect of actually attaching the paper has not been simulated in this paper. Therefore, the result is not an exact match to the result produced through the man-made colored paper mosaic effect. Future works should concentrate on man-made effects such as wrapped and overlapped paper caused in the rendering process. By researching a variety of phenomena in authentic work, these physical phenomena will be simulated in the future. And while approximating edge map to geometrical edge, approximated edges sometimes do not fit completely around the borderline between two adjacent segmented layers. These inconsistencies don't affect the rendered image a lot, but will be considered. Also, the shapes of the attached paper used for the simulation should also be diverse for various mosaic effects.

\section{Acknowledgement}

This work was partially supported by the Chung-Ang University Special Research Grants No.961173 in 1996

\section{References}

1. Aaron Hertzmann: Paintery Rendering with Curtved Brush Strokes of Multiple Sizes, In SIGGRAPH 98 Proceedings, (1998) 453-460

2. Alan Watt: 3D Computer Graphics, Addison Wesley (2000) 
3. Cassidy J. Curtis: Loose and Sketchy Animation, In SIGGRAPH'98 Abstracts and Applications (1998) 317

4. Daniel Teece: Three Dimensional Interactive Non PhotoRealistic Rendering, PhD. thesis, University of Sheffield, England (1998)

5. A. Fournier, D. Fussell, and L. Carpenter: Computer Rendering ofStochastic Models, Communication of ACM, Vol.25, No.6 (1982)371-384

6. Gonzalez, Woods: Digital Image Processing, Addison Wesley (1993)

7. Stuart Green: Non-Photorealistic Rendering, SIGGRAPH'99 Course Note\#17 (1999)

8. Alejo Hausner: Simulating Decorative Mosaics, In SIGGRAPH'2001 Proceedings (2001) 573-580

9. Mark de Berg, M. V. Kerveld, M. Overmars and O. Schwarzkopf: Computational Geometry Algorithms and Applications, Springer-Verlag (1997) 145-161

10. Michael F. Barnsley: Fractals Everywhere. 2nd Edition, AP Professional (1993)

11. Miyata, K.: A method of generating stone wall patterns, In SIGGRAPH'90 Proceedings (1990) 387-394

12. Sang Hyun Seo, Young Sub Park, Sung Ye Kim and Kyung Hyun Yoon: Colored Paper Mosaic Rendering, In SIGGRAPH'2001 Sketchy and Applications (2001) 156

13. Young Sub Park, Sung Ye Kim, Chung Woon Cho and Kyung Hyun Yoon: Manual Color Paper Mosaic Technique, Journal of Computer Graphics Vol 4 (2002) 123130, In Korean
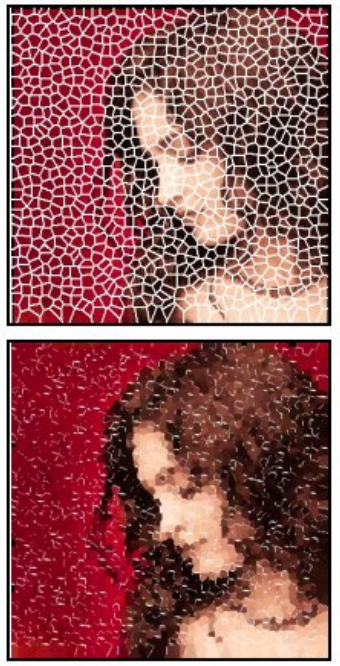

(a) Voronoi diagram
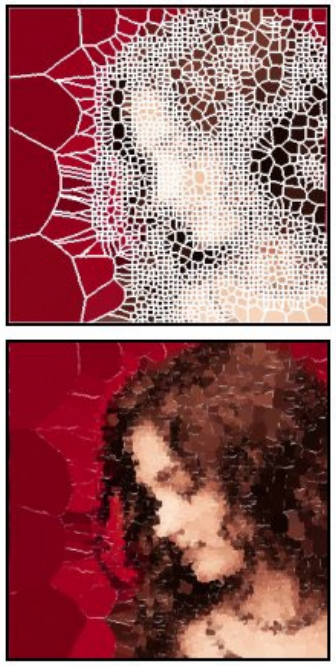

(b) Voronoi diagram by quad tree
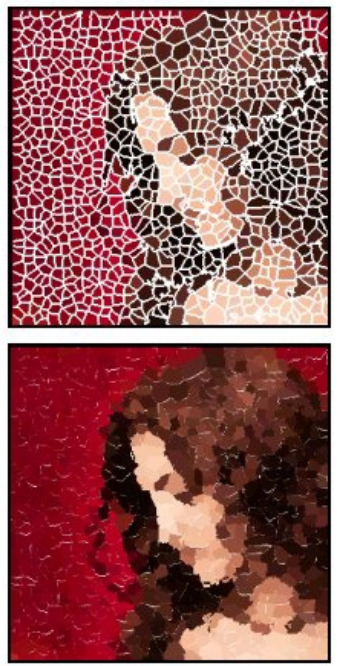

(c) Voronoi diagram on each segmented layer

Fig. 7. Result images 1: Voronoi diagram and images of colored paper mosaic rendering 